\title{
Equidad en el apoyo social recíproco y satisfacción vital en personas mayores
}

Equity in the Reciprocal Social Support and Life Satisfaction in Older People

Recepción: Octubre , 17, 2013 | Aprobación: Enero , 23, 2017

\author{
Belén Bueno Martínez ${ }^{\mathrm{a}}$ \\ Universidad de Salamanca, España \\ José Buz \\ Universidad de Salamanca, España \\ Ana Belén Navarro \\ Universidad de Salamanca, España \\ LARA LÓPEZ-MARTÍN \\ Centro de Servicios Sociales La Fuencisla, España
}

a Autor de correspondencia. Correo electrónico: bbueno@usal.es

Para citar este artículo: Bueno, B., Buz, J., Navarro, A.B., \& López-Martín, L. (2017). Equidad en el apoyo social recíproco y satisfacción vital en personas mayores. Universitas Psychologica, 16(1), 1-13. http://d x.doi.org/10.11144/Javeriana.upsy16-1.easr

\begin{abstract}
RESUMEN
Se examinó la asociación entre equidad en el apoyo social y bienestar en personas mayores españolas (Rango 60-98, $\mathrm{M}=74.04 ; \mathrm{DE}=7.75 ; \mathrm{N}$ $=585)$. Se obtuvo información sobre variables sociodemográficas y de autonomía funcional, miembros activos de varias redes de apoyo, apoyo dado y recibido emocional e instrumental y satisfacción vital. La equidad caracteriza la mayoría de los intercambios de apoyo de los mayores con sus redes familiares y sociales. Los intercambios recíprocos equitativos y ser proveedor activo en el intercambio, se relacionan positivamente con la satisfacción vital en la vejez. La naturaleza del apoyo emocional o instrumental modifica esos resultados. La Teoría de la Equidad obtiene más evidencia empírica que la Teoría del Intercambio Social.
\end{abstract}

\section{Palabras clave}

Apoyo social; equidad; personas mayores; reciprocidad; satisfacción vital.

\section{ABSTRACT}

The association between perceived equity in social support and wellbeing was examined in older people (Age 60-98, $\mathrm{M}=74.11 ; \mathrm{SD}=7.92 ; \mathrm{N}$ $=585)$. Data were from a study at Salamanca (Spain). Information was obtained about socio-demographic and functional autonomy variables, active members from several support networks, given and received support of instrumental and emotional type, and a life satisfaction index. The equity defines the majority of support exchanges that elderly maintain with their kin and non-kin networks. Balanced reciprocal exchanges, besides the capacity to be an active provider in exchange, are positively related to life satisfaction in old age. The nature of the emotional or instrumental support modifies these results. Equity Theory gets more empirical evidence than Social Exchange Theory.

Keywords

aged; equity; life satisfaction; reciprocity; social support. 
Diversos estudios muestran que las personas casadas experimentan más felicidad y menos estrés psicológico que las personas que no lo están (Lee \& Ono, 2012). La cantidad y la calidad del apoyo dado y recibido en el contexto del matrimonio y fuera de él tienen un notable impacto sobre el bienestar subjetivo. Sin embargo, muy pocos estudios han tenido en cuenta la calidad de las relaciones de apoyo de las personas mayores casadas y no casadas (Hagedoorn et al., 2006). Las evidencias sobre la validez de las teorías más utilizadas para explicar la vinculación entre las relaciones de apoyo y el bienestar ofrecen resultados inconsistentes en personas mayores (Li, Fok, \& Funk, 2011; Lowenstein, Katz, \& Gur-Yaish, 2007; Tsuboi, Hiray, \& Kondo, 2016).

La Teoría del Intercambio Social ([SET], Dowd, 1975) asume que las personas buscan maximizar los beneficios en las relaciones sociales y minimizar los costos, de modo que quienes reciben más de lo que dan experimentan los niveles más altos de bienestar. La Teoría de la Equidad ([ET], Walster, Walster, \& Berscheid, 1978) defiende que los niveles de satisfacción más elevados se asocian a las relaciones de ayuda equitativas. Por el contrario, quienes reciben más apoyo del que dan se sienten psicológicamente mal, porque no responden a la norma social o a sus estándares de reciprocidad, y quienes dan más de lo que reciben, obtienen niveles de bienestar bajos al percibir que su esfuerzo no es correspondido.

Los hallazgos empíricos basados en la SET y en la ET en la vejez no son consistentes. Aunque se han encontrado correlaciones positivas entre dar y recibir ayuda (Grundy, 2005; van Tilburg, \& Broese van Groenou, 2002), también existen trabajos que no apoyan ninguna de las dos teorías (Kim, Hisata, Kai, \& Lee, 2000). Dado que las evidencias sobre la validez de estas teorías suelen centrarse de manera general en las relaciones familiares y sociales, en este trabajo se ponen a prueba sus postulados, considerando el intercambio de apoyo entre miembros específicos de la red social del individuo.
En el caso del matrimonio, se sabe que la disminución de la red social en la vejez convierte el apoyo y la compañía del cónyuge en un elemento clave del bienestar (AhmedMohamed, Fernández-Mayoralas, Rojo-Pérez, Forjaz, \& Martínez-Martín, 2013; MeléndezMoral, Tomás-Miguel, \& Navarro-Pardo, 2007). Las relaciones con los hijos adultos son otro factor clave del bienestar (Fernández-Ballesteros, 2002; Nyqvist, Forsman, Giuntoli, \& Cattan, 2013), aunque se ha resaltado su complejidad porque combinan recursos y necesidades de ambas partes (Ang \& Malhotra, 2016; Lee, Lyu, Lee, \& Burr, 2013). Las relaciones con otros familiares adultos, principalmente los hermanos, apenas han sido estudiadas (Thomas, 2010; Voorpostel, Lippe, Dykstra, \& Flap, 2007). Fuera del entorno familiar, los amigos y vecinos constituyen alrededor de la mitad de la red social más activa, pero se sabe poco sobre su impacto en el bienestar de las personas mayores (De Jong Gierveld, \& Perlman, 2006; Li, Ji, \& Chen, 2014).

Hasta la actualidad, existen muy pocos estudios realizados con personas mayores españolas sobre las relaciones de apoyo entre los miembros de la red social y su impacto sobre el bienestar subjetivo. Únicamente, en el estudio OASIS se han analizado las relaciones intergeneracionales entre padres mayores $\mathrm{e}$ hijos adultos, incluyendo a España entre los países investigados (Lowenstein \& Daatland, 2006). España es un país caracterizado por la importancia de las relaciones familiares donde las normas de responsabilidad filial son elevadas, lo que resulta congruente con patrones de intercambio equitativo entre padres mayores e hijos adultos (Lowenstein, Katz, \& GurYaish, 2008). Desconocemos si las relaciones de intercambio equitativo predominan o no en otras relaciones interpersonales importantes para el bienestar de los mayores. Por ello, en este trabajo se identificó el perfil de reciprocidad de las relaciones de apoyo y se examinó la relación entre los intercambios de apoyo y la satisfacción vital en personas mayores españolas, teniendo en cuenta el estado civil y el tipo de red social. Para ello, se midió la reciprocidad del apoyo instrumental 
y emocional en las relaciones con el cónyuge, los hijos, otros familiares, los amigos y los vecinos.

Los objetivos del estudio fueron: (1) describir los niveles de reciprocidad en las redes sociales de personas mayores españolas en función de su estado civil y (2) examinar desde la SET y la ET el impacto de la reciprocidad sobre la satisfacción vital en la vejez, considerando distintos tipos de apoyo (instrumental y emocional) y diferentes redes sociales.

Se espera que predominen las relaciones recíprocas en los diferentes estados civiles y situaciones parentales de las personas mayores, aunque la proporción relativa variará dependiendo del tipo de red social (Hipótesis 1). De acuerdo con la ET, se espera que los intercambios equilibrados se relacionen positivamente con la satisfacción vital de las personas mayores en las diferentes relaciones interpersonales que mantienen (Hipótesis 2a); siguiendo a la SET, se espera que los intercambios en los que se recibe más de lo que se da se asocien con niveles elevados de satisfacción vital (Hipótesis 2b).

\section{Método}

\section{Participantes y procedimiento}

En esta investigación, participaron 720 personas mayores de 60 años, no institucionalizadas, residentes en Salamanca y seleccionadas mediante un muestreo estratificado por edad y sexo, por afijación simple, a partir de los datos del Padrón Municipal. De la muestra total, un $15.69 \%$ no finalizó el cuestionario; el $12.08 \%$ por decisión propia y el $3.61 \%$ por dificultades debido a problemas auditivos y/ o visuales. El $3.05 \%$ fue excluido por presentar sospechas de deterioro cognitivo según el MiniMental State Examination (MMSE) (Folstein, Folstein, \& McHugh, 1975) (MMSE < 23). En consecuencia, para este estudio se examinaron los datos de 585 participantes $(\mathrm{M}=74.11, \mathrm{DE}=$ 7.92, Rango $=60$-98). La Tabla 1 muestra sus características sociodemográficas.
Tras explicar a los participantes el objetivo del estudio y obtener el consentimiento, se concertó una cita o se procedió a aplicar el cuestionario. Se realizó una aplicación piloto a 20 personas mayores de diferentes edades y sexos, para identificar posibles dificultades con los ítems y/o formatos de respuesta, así como con el proceso de administración, lo que permitió modificar el cuestionario, maximizando la correcta interpretabilidad de los enunciados y la adecuación del proceso de administración. La recogida de datos con el cuestionario final fue realizada por entrevistadores entrenados, en el domicilio de los ancianos y sin terceras personas. La duración de la entrevista fue de aproximadamente 1 hora. Durante la aplicación del cuestionario se garantizó el anonimato y confidencialidad en la recogida y el tratamiento de los datos. La participación fue voluntaria y no remunerada.

\section{TABLA 1}

Características sociodemográficas de la muestra

\begin{tabular}{ll} 
Variable & Porcentaje \\
Edad (años) & \\
$60-74$ años & $49.9(n=292)$ \\
$75+$ años & $50.1(n=293)$ \\
Rango (60-98); $M=74.04 ; D E=7.75$ & \\
\hline Sexo (\%) & \\
Hombre & $46.5(n=272)$ \\
Mujer & $53.5(n=313)$ \\
\hline Estado Civil (\%) & \\
Casados & $56.9(n=333)$ \\
Viudos & $33.3(n=195)$ \\
Solteros & $7.5(n=44)$ \\
Separados/Divorciados & $2.2(n=13)$ \\
\hline Nivel Educativo (\%) & \\
Analfabeto & $4.4(n=26)$ \\
Sabe leer y escribir & $14.7(n=86)$ \\
Ha ido a la escuela & $73.5(n=430)$ \\
Ha ido a la universidad & $7.4(n=43)$ \\
\hline Tiene Hijos (\%) & $13.8(n=81)$ \\
No & $86.2(n=504)$ \\
Sí & \\
\hline No De Hijos & \\
Rango (1-11); $M=2.91 ; D E=1.32$ & \\
\hline Con Quien Vive (\%) & \\
Solo & $26.8(n=157)$ \\
Con otras personas & $2.6(n=15)$ \\
Sin cónyuge pero con hijos & $9.6(n=56)$ \\
Con cónyuge y sin hijos & $38.8(n=227)$ \\
Con cónyuge e hijos & $16.9(n=99)$ \\
Otras situaciones & $5.3(n=31)$ \\
\hline &
\end{tabular}

Nota: $M=$ media; $D E=$ desviación estándar. Fuente: elaboración propia. 


\section{Instrumentos}

La selección de instrumentos se hizo basándose en una investigación transcultural más amplia iniciada previamente (Sánchez, de Jong Gierveld, $\&$ Buz, 2014) que queda fuera del alcance de este estudio. Por ello, algunos instrumentos y procedimientos siguen la metodología empleada en el Longitudinal Aging Study Amsterdam (LASA) (Deeg, Beekman, Kriegsman, \& Westendorp-de Serière, 1998). Para este estudio, únicamente se utilizaron las variables sociodemográficas, de salud, de apoyo social y de satisfacción con la vida. Las variables sociodemográficas medidas fueron sexo, edad, estado civil, nivel educativo, condiciones de vida, número de personas en el hogar y número de hijos.

Se midió la autonomía funcional mediante la escala de actividades de la vida diaria (e. g., subir y bajar escaleras) desarrollada en el LASA, que demostró tener adecuadas garantías psicométricas y buen poder predictivo de la vida social activa y participativa. Las respuestas se daban en una escala tipo Likert de cinco puntos (desde 1 = la actividad no se puede hacer hasta 5 $=$ se puede realizar sin ningún tipo de ayuda). La puntuación final es la media de todos los ítems. La fiabilidad fue alta $(\alpha=0.93)$.

Se calculó el apoyo social, utilizando el procedimiento seguido por Boerner y Reinhart (2003) de evaluar la red social centrada en la persona, basándose en dominios específicos de la red social (cónyuge, hijos, hermanos, amigos y vecinos). Para ello, se pidió a los participantes que identificaran con las iniciales a cada uno de los miembros de su red social familiar (cónyuge, hijos y hermanos) y no familiar (amigos y vecinos). Posteriormente, siguiendo a Silverstein, Conroy, Wang, Giarrusso y Bengtson (2002), se preguntó por el apoyo social dado y recibido (en ambos casos instrumental y emocional) de cada uno de los miembros de la red social. El apoyo dado de tipo instrumental se midió con el ítem "iCuánto ha ayudado usted en tareas cotidianas (compras, limpiar, etc.) a D/Dña X en el último año?" y el emocional con el ítem "iCuánto ha hablado a D/Dña $X$ de sus sentimientos o problemas personales en el último año?". El mismo procedimiento se siguió para el apoyo recibido. Las respuestas se daban en una escala que iba de $1=$ Nunca a $4=$ A menudo. Se calculó el apoyo dado y recibido, tanto instrumental como emocional, obteniendo la media en cada grupo de la red social.

Siguiendo a van Tilburg (1992), se valoró un índice de reciprocidad, a partir de las medidas de apoyo dado y recibido de cada grupo de la red social y de cada tipo de apoyo. Para ello, se evaluó el cociente entre la cantidad de apoyo recibido $\mathrm{y}$ dado en apoyo instrumental y emocional. Posteriormente, los valores se transformaron en una variable de tres categorías $(<1=$ infrabeneficiado, $>1=$ sobre-beneficiado y $1=$ equitativo)

Se midió el bienestar subjetivo con la versión española de la Escala de Satisfacción con la Vida (Diener, Emmons, Larsen, \& Griffin, 1985) proporcionada por el autor (Diener, 2009). Consta de 5 enunciados sobre aspectos importantes de la vida, habiéndose valorado el grado de acuerdo en una escala tipo Likert (desde 1 = Totalmente en desacuerdo hasta $4=$ Totalmente de acuerdo). La puntuación total se obtiene sumando las puntuaciones de los cinco ítems. Una puntuación más alta significa mayor satisfacción con la vida. La fiabilidad obtenida fue de 0.85 .

Se valoró el estado de ánimo con la versión breve de la escala de depresión geriátrica ([GDS-8], Buz, 1996). Las respuestas se daban en una escala dicotómica $(0=$ no, $1=$ sí). La puntuación total oscilaba entre 0 y 8 puntos. Una mayor puntuación indicaba mayor presencia de síntomas depresivos. Estudios en población comunitaria española han mostrado adecuadas propiedades psicométricas (Buz, Mayoral, Bueno, \& Vega, 2004). La fiabilidad se estimó en 0.93. En la Tabla 2, se presentan los descriptivos de las variables anteriores, exceptuando las de tipo sociodemográfico y condiciones de vida. 
TABLA 2

Descriptivos de las variables de apoyo y de satisfacción vital $(\mathrm{N}=585)$

\begin{tabular}{lll} 
Variable & $M$ & $D E$ \\
\hline Estado funcional & 4.27 & 1.06 \\
\hline $\begin{array}{l}\text { Apoyo recibido } \\
\text { Instrumental... }\end{array}$ & & \\
del cónyuge & 3.47 & 0.94 \\
de los hijos & 2.7 & 0.95 \\
de otros familiares & 2.09 & 0.92 \\
de los amigos & 1.92 & 1.01 \\
$\quad$ de los vecinos & 1.94 & 0.94 \\
Emocional... & & \\
del cónyuge & 3.63 & 0.6 \\
de los hijos & 2.98 & 0.8 \\
de otros familiares & 2.35 & 0.9 \\
de los amigos & 2.92 & 0.8 \\
de los vecinos & 2.21 & 0.9 \\
& & \\
\hline Apoyo dado & & \\
Instrumental... & & 0.86 \\
al cónyuge & 3.47 & 1.02 \\
$\quad$ a los hijos & 2.52 & 0.92 \\
$\quad$ a otros familiares & 1.9 & 1 \\
a los amigos & 1.9 & 0.93 \\
a los vecinos & 1.88 & \\
Emocional... & & 0.7 \\
al cónyuge & 3.61 & 0.89 \\
a los hijos & 2.89 & 0.92 \\
a otros familiares & 2.33 & 0.87 \\
a los amigos & 2.88 & 1 \\
\hline a los vecinos & 2.24 & 2.46 \\
\hline Escala de satisfacción con la vida & 14.15 & \\
\hline & & \\
\hline
\end{tabular}

Nota: $M=$ media; $D E=$ desviación estándar. Fuente: elaboración propia.

\section{Análisis estadísticos}

Para describir el perfil de reciprocidad de las relaciones de apoyo, se calcularon los porcentajes de relaciones de apoyo equitativas y no equitativas, en cada una de las redes sociales de las personas mayores casadas, solteras y viudas/ separadas-divorciadas. Para examinar la relación entre el estado civil con respecto a las situaciones de reciprocidad en las relaciones (Hipótesis 1), se realizaron pruebas de chi-cuadrado. Para comprobar las diferencias en satisfacción vital entre los intercambios de ayuda equitativos y no equitativos en las distintas redes de apoyo y si se producían en el sentido pronosticado por la ET o por la SET (Hipótesis 2a y 2b), se efectuaron las ANOVA, controlando el estado funcional y tomando como factor intersujetos las medidas de reciprocidad en el apoyo instrumental y emocional en cada grupo de la red social.

\section{Resultados}

En relación con la Hipótesis 1, la Tabla 3 muestra los porcentajes de equidad y no equidad en cada una de las dimensiones evaluadas de las redes de apoyo.

\section{TABLA 3}

Porcentajes de tipos de reciprocidad en las relaciones de apoyo en función del estado civil y parental (entre paréntesis, $n$ correspondiente a cada porcentaje)

\begin{tabular}{|c|c|c|c|c|}
\hline & & $\begin{array}{l}\text { Casados con hijos } \\
(n=325)\end{array}$ & $\begin{array}{l}\text { No casados con hijos } \\
(n=189)\end{array}$ & $\begin{array}{l}\text { No casados sin hijos } \\
(n=44)\end{array}$ \\
\hline \multicolumn{5}{|l|}{ Cónyuge } \\
\hline \multirow{2}{*}{ Da más } & Instrumental & $15.5(50)$ & & \\
\hline & Emocional & $\begin{array}{ll}6.5 \quad(21) \\
\end{array}$ & & \\
\hline \multirow{2}{*}{ Equidad } & Instrumental & $65 \quad(211)$ & & \\
\hline & Emocional & 85.5 (278) & & \\
\hline \multirow{2}{*}{ Recibe mís } & Instrumental & $19.5(64)$ & & \\
\hline & Emocional & $8 \quad(26)$ & & \\
\hline \multicolumn{5}{|l|}{ Hijos } \\
\hline \multirow{2}{*}{ Da más } & Instrumental & $23.3(76)$ & 14.1 (27) & \\
\hline & Emocional & $14.8(48)$ & $13 \quad(25)$ & \\
\hline \multirow{2}{*}{ Equidad } & Instrumental & 46.9 (152) & $44.9(85)$ & \\
\hline & Emocional & $61.6(200)$ & $58.4(110)$ & \\
\hline \multirow{2}{*}{ Recibe mis } & Instrumental & $29.8(97)$ & 41.1 (77) & \\
\hline & Emocional & $23.6(77)$ & $28.6(54)$ & \\
\hline \multicolumn{5}{|l|}{ Otra familia } \\
\hline \multirow{2}{*}{ Da más } & Instrumental & $11.5 \quad(37)$ & $10.8(20)$ & $21.6(10)$ \\
\hline & Emocional & $9.6 \quad(31)$ & $8.2(15)$ & $16.2(7)$ \\
\hline \multirow{2}{*}{ Equidad } & Instrumental & $80 \quad(260)$ & $79.1(150)$ & 62.2 (27) \\
\hline & Emocional & 83.4 (271) & $77.2(146)$ & $73 \quad(32)$ \\
\hline \multirow{2}{*}{ Recibe más } & Instrumental & $8.5 \quad(28)$ & $10.1(19)$ & $16.2(7)$ \\
\hline & Emocional & $\begin{array}{ll}7.0 & (23) \\
\end{array}$ & $14.6(28)$ & $10.8(5)$ \\
\hline \multicolumn{5}{|l|}{ Amigos } \\
\hline \multirow{2}{*}{ Da más } & Instrumental & $6.1(20)$ & $11.6(22)$ & $12.5(5)$ \\
\hline & Emocional & $6.1 \quad(20)$ & $8.8(17)$ & $7.5 \quad(3)$ \\
\hline \multirow{3}{*}{ Equidad } & Instrumental & $86.5(281)$ & 73.4 (139) & $\begin{array}{ll}75 & (33) \\
\end{array}$ \\
\hline & Emocional & $\begin{array}{ll}85 & (276)\end{array}$ & 80.3 (152) & $72.5(32)$ \\
\hline & Instrumental & 7.4 (24) & 15 (28) & $12.5(5)$ \\
\hline Recibe mis & Emocional & 8.9 (29) & $10.9(20)$ & $20 \quad(9)$ \\
\hline Vecinos & Instrumental & $9.3(30)$ & $10.5(20)$ & $6.7 \quad(3)$ \\
\hline \multirow[t]{2}{*}{ Da más } & Emocional & $8.2(27)$ & 8.2 (16) & $16.7(7)$ \\
\hline & Instrumental & $78.6(256)$ & 70.5 (133) & $86.7(38)$ \\
\hline \multirow[t]{2}{*}{ Equidad } & Emocional & $87.4(284)$ & 80.2 (151) & $80 \quad(35)$ \\
\hline & Instrumental & 12.1 (39) & $\begin{array}{ll}19 & (36)\end{array}$ & $6.7(3)$ \\
\hline Recibe mis & Emocional & & & \\
\hline
\end{tabular}

Nota: El grupo de personas casadas sin hijos es eliminado por poseer un $n$ muy pequeño $(n=8)$. Fuente: elaboración propia

Las relaciones equitativas predominan en las distintas redes de apoyo, con las cifras más elevadas de equidad en los intercambios con hermanos, amigos y vecinos, tanto instrumentales como emocionales, seguidos de las relaciones de apoyo con el cónyuge y con los hijos. A partir de los análisis de contingencia realizados, se obtuvo que la reciprocidad era independiente del estado civil en las relaciones de apoyo emocional con los hijos $\chi_{\chi}^{2}=1.61, g l=2$, $n=490, p=0.447)$, con los amigos $\left(\chi^{2}=5.702\right.$, $g l=4, n=433, p=0.223$ ), en las relaciones con los hermanos, tanto instrumentales $\chi^{2}=6.492$, 
$g l=4, n=465, p=0.165)$ como emocionales $\left.\chi_{\chi}^{2}=8.532, g l=4, n=466, p=0.074\right) \mathrm{y}$ en las relaciones con los vecinos, instrumentales $\left(\chi^{2}=4.848, g l=4, n=334, p=0.303\right) y$ emocionales $\left(\chi^{2}=8.685, g l=4, n=333, p=\right.$ 0.069). Únicamente, se encontró una relación de dependencia entre la reciprocidad y el estado civil en las relaciones de ayuda instrumental con los hijos $\left(\chi^{2}=9.324, g l=4, n=490, p=0.009\right)$ y con los amigos $\left(\chi^{2}=11.273, g l=4, n=433\right.$, $p=0.024)$.

El análisis de los residuos tipificados corregidos indicó que, en los intercambios de apoyo instrumental con sus hijos, había más casados de los esperados infrabeneficiados y menos sobrebeneficiados, patrón contrario al obtenido entre las personas viudas y separadas/divorciadas. En las relaciones de ayuda instrumental con los amigos, había menos intercambios equitativos de los esperados y más intercambios sobrebeneficiados entre los viudos y separados/ divorciados, mientras que entre los casados había más intercambios equitativos de los esperados y menos intercambios no equitativos.

En relación con las Hipótesis 2a y 2b, se examinó qué situaciones de reciprocidad producían mayores niveles de satisfacción, considerando los miembros de la red social familiar y no familiar- y el tipo de apoyo. Se observó que en el caso del cónyuge, los niveles de satisfacción vital eran significativamente más altos, $F(2,252)=3.167, p<0.05$, cuando el intercambio de ayuda instrumental era equitativo que cuando no lo era (Tabla 4A). Sin embargo, no ocurrió lo mismo con el apoyo emocional $F(2$, 253) $=0.573, p=0.565$.

Se encontró la situación contraria respecto a los hijos. Mientras que en las relaciones de ayuda instrumental, el nivel de satisfacción vital era similar en los intercambios equitativos y no equitativos, $F(2,394)=1.981, p=0.139$, en las relaciones de apoyo emocional los intercambios equitativos producen los niveles más elevados de bienestar, $F(2,393)=3.375, p=0.03$.

Con los hermanos, las relaciones de ayuda equitativas de tipo instrumental eran las que producían mayor satisfacción vital en las personas mayores, $F(2,376)=7.728, p=0.001$, seguido de las situaciones en que se da más de lo que se recibe. Sin embargo, cuando se trata de apoyo emocional, los intercambios equitativos y no equitativos producen niveles similares de bienestar, $F(2,377)=0.007, p=0.993$.

\section{TABLA 4A}

Medias de satisfacción vital según la situación de reciprocidad para cada grupo de la red y tipo de apoyo, controlando el estado funcional

\begin{tabular}{|c|c|c|c|c|c|c|}
\hline & \multicolumn{2}{|c|}{$\begin{array}{l}\text { Cónyuge } \\
(\mathrm{n}=262)\end{array}$} & \multicolumn{2}{|c|}{$\begin{array}{c}\text { Hijos } \\
(\mathrm{n}=403)\end{array}$} & \multicolumn{2}{|c|}{$\begin{array}{l}\text { Hermanos } \\
(\mathrm{n}=385)\end{array}$} \\
\hline & Instrumental & Emocional & Instrumental & Emocional & Instrumental & Emocional \\
\hline $\begin{array}{l}\text { Equidad } \\
\text { Lidis }\end{array}$ & $14.82^{\mathrm{a}}$ & 14.60 & $\begin{array}{l}14.50 \\
14.34\end{array}$ & $14.53^{\mathrm{a}}$ & $14.35^{\mathrm{a}}$ & 14.20 \\
\hline Da más de lo que recibe & $14.26^{b}$ & 14.41 & 14.34 & $13.88^{b}$ & $14.19^{b}$ & 14.19 \\
\hline Recibe más de lo que da & $13.88^{b}$ & 14.10 & 13.82 & $13.76^{\mathrm{b}}$ & $12.53^{\mathrm{c}}$ & 14.05 \\
\hline $\begin{array}{c}p \\
\eta^{2}\end{array}$ & $\begin{array}{l}0.044 \\
0.025\end{array}$ & $\begin{array}{l}n s \\
0.005\end{array}$ & $\begin{array}{c}n s \\
0.010\end{array}$ & $\begin{array}{l}0.035 \\
0.017\end{array}$ & $\begin{array}{l}0.001 \\
0.039\end{array}$ & $\begin{array}{l}n s \\
0\end{array}$ \\
\hline
\end{tabular}

$$
\begin{gathered}
\text { Nota: } n s=\text { no significativo; } \\
\eta^{2}=\text { tamaño del efecto. } \\
\text { a b c Las medias con distinto subíndice son } \\
\text { significativamente distintas entre sí en las } \\
\text { comparaciones con pares al nivel } p<.05 \text { (pruebas } \\
\text { F y contrastes Scheffé a posteriori en ANOVAS). } \\
\text { Fuente: elaboración propia }
\end{gathered}
$$

Por lo que se refiere a la red no familiar (Tabla 4B), en el caso de los amigos, los intercambios equitativos y aquellos en los que se daba más de lo que se recibía, producían los niveles de satisfacción vital más elevados, tanto para la ayuda instrumental, $\mathrm{F}(2,348)$ $=3.236, p=0.041$, como emocional, $\mathrm{F}(2$, $348)=3.434, p=0.033$. Sin embargo, con los vecinos, no se encontraron diferencias significativas en satisfacción vital en función de que los intercambios instrumentales y emocionales fueran o no equitativos, $\mathrm{F}(2,264)=$ $1.934, p=0.147$ y $\mathrm{F}(2,263)=0.821, p=0.441$, respectivamente. 
TABLA 4B

Medias de satisfacción vital según la situación de reciprocidad para cada grupo de la red no familiar y tipo de apoyo, controlando el estado funcional

\begin{tabular}{lccccc} 
& \multicolumn{2}{c}{$\begin{array}{c}\text { Amigos } \\
(\mathrm{n}=357)\end{array}$} & & \multicolumn{2}{c}{$\begin{array}{c}\text { Vecinos } \\
(\mathrm{n}=272)\end{array}$} \\
\cline { 2 - 3 } \cline { 5 - 6 } \cline { 5 - 6 } & Instrumental & Emocional & & & \multicolumn{2}{c}{} \\
\hline Equidad & $14.21^{\mathrm{a}}$ & $14.18^{\mathrm{a}}$ & & 14.16 & 14.07 \\
Da más de lo que recibe & $14.47^{\mathrm{a}}$ & $14.68^{\mathrm{a}}$ & & 14.00 & 13.40 \\
Recibe más de lo que da & $13.16^{\mathrm{b}}$ & $13.19^{\mathrm{b}}$ & & 13.22 & 13.94 \\
$p$ & 0.041 & 0.033 & & $n \mathrm{~s}$ & $n s$ \\
$\eta^{2}$ & 0.018 & 0.019 & & 0.014 & 0.006 \\
\hline
\end{tabular}

Nota: $n s=$ no significativo;

$\eta^{2}=$ tamaño del efecto.

a b Las medias con distinto subíndice son significativamente distintas entre sí en las comparaciones por pares al nivel $p<0.05$ (pruebas $F$ y contrastes Scheffé a posteriori en ANOVAS). Fuente: elaboración propia.

\section{Discusión}

El presente estudio realiza varias contribuciones distintivas a la bibliografía existente sobre reciprocidad en el apoyo social y bienestar en la vejez. Primero, se identificaron los intercambios equitativos y no equitativos de las personas mayores de nuestro entorno, en las diferentes redes familiares y sociales que mantienen, así como su perfil de reciprocidad en función del estado civil y de la situación parental. Además, se sometieron a prueba los postulados de la SET y de la ET para explicar las diferencias en satisfacción vital en función del intercambio con las redes sociales más relevantes.

En apoyo a la Hipótesis 1, las personas mayores estudiadas mantienen mayoritariamente intercambios recíprocos equilibrados. La proporción relativa de intercambios equitativos y no equitativos se mantiene en las redes de apoyo emocional en los diferentes estados civiles y situaciones parentales, así como en los intercambios de ayuda instrumental con los hermanos y con los vecinos. Estos hallazgos ponen de relieve que los intercambios de apoyo dentro y fuera de la familia tienen en la reciprocidad un eje fundamental (Armi, Guilley, \& Lalive d'Epinay, 2008; Johar, Maruyama, \& Nakamura, 2015; McMunn, Nazroo, Wahrendorf, Breeze, \& Zaninotto, 2009; Shaw, 2005) y van en la línea de los trabajos que defienden el papel de la reciprocidad en la continuidad de las relaciones (Fyrand, 2010). A lo largo de la vida, la reciprocidad igualaría los desequilibrios de los intercambios de ayuda presentes y anteriores, asegurando la continuidad en las relaciones íntimas, particularmente entre esposos y entre padres mayores e hijos adultos (Ikkink \& van Tilburg, 1999). Además, la reciprocidad parece tener un papel fundamental en la decisión de los hijos adultos de volver a convivir con los padres mayores, en caso de necesidad por parte de alguno de ellos (Johar et al., 2015). Entre amigos, la independencia se mantiene sobre la base de relaciones recíprocas equilibradas. Y en relaciones más periféricas y más susceptibles a la finalización, con hermanos y vecinos, la reciprocidad en todo tipo de ayuda puede ser la clave del mantenimiento de la relación.

Situaciones vitales, como la viudez y el estar separado/divorciado, pueden generar demandas desequilibradas de ayuda instrumental con los hijos y con los amigos en comparación con quienes siguen disponiendo del apoyo del cónyuge. Sin embargo, las personas mayores que nunca se han casado mantienen mayoritariamente intercambios equitativos emocionales e instrumentales con sus hermanos, sus amigos y sus vecinos (DePaulo \& Morris, 2005).

Al explorar el impacto de la ayuda recíproca sobre la satisfacción vital desde los postulados de la SET y de la ET, se observó que los resultados apoyaban parcialmente los postulados de la ET, aunque los tamaños del efecto son pequeños. En ningún caso se obtuvieron resultados que apoyaran la SET. El papel del tipo y de la fuente de apoyo parece haber sido muy importante.

La distinción entre apoyo instrumental y emocional con el cónyuge arroja diferencias en la satisfacción vital generada por la presencia o no de equidad en la relación. Mientras las relaciones equitativas instrumentales que las personas mayores mantienen con su cónyuge influyen positivamente sobre su satisfacción vital, no sucede lo mismo con las relaciones equitativas emocionales. El primer resultado apoya la ET y va en la línea de los obtenidos por otros 
investigadores. Sin tener en cuenta la naturaleza del apoyo intercambiado, Hagedoorn et al. (2006) encontraron que, al envejecer, los casados que experimentaban mayores niveles de bienestar eran quienes mantenían relaciones de ayuda equitativas con su pareja. Con el envejecimiento, el deterioro de la salud va acompañado de mayores niveles de dependencia en tareas cotidianas. Esta necesidad de ayuda suele ser proporcionada por las personas del entorno más inmediato (Fingerman, Kim, Tennant, Birditt, $\&$ Zarit, 2015). En culturas colectivistas, como los países mediterráneos y Asia, el apoyo para las personas más vulnerables procede de la familia (cónyuge e hijos) y los amigos (AhmedMohamed et al., 2013; Lu, Liu, \& Lou, 2016). Este tipo de apoyo suele estar relacionado con mayores niveles de bienestar, ya que responde a expectativas culturales y permite adaptar la vida diaria al declive funcional (Ang \& Malhotra, 2016; Leopold \& Raab, 2013). La ausencia de diferencias en satisfacción vital entre los intercambios emocionales equitativos y no equitativos con el cónyuge va en contra de la ET y de los resultados de los estudios previos comentados. Los resultados del presente trabajo indican que el que la persona mayor dé más, dé menos o dé la misma cantidad de apoyo emocional al cónyuge, no influye en sus niveles de bienestar subjetivo. Es posible que la larga historia compartida de intimidad a lo largo de toda una vida de casados, haga que el balance entre lo que se da y se recibe emocionalmente del cónyuge pierda valor en la vejez. Futuros estudios longitudinales podrían investigar esta hipótesis relacionada con los cambios en la percepción del apoyo dependiendo de su naturaleza, y examinar su impacto sobre el bienestar subjetivo. Otras posibles hipótesis estarían relacionadas con la existencia de mecanismos fisiológicos distintos para hombres y mujeres en la explicación de la relación entre la reciprocidad de ayuda y el bienestar. En concreto, parece que la presencia de hormonas reproductivas, como la oxitocina, produce emociones positivas cuando la persona se siente querida y apoyada (Hendrix, Baumgartner, Kirschbaum, \& Ehlert, 2003).
En un estudio reciente sobre la relación entre fuentes y tipos de apoyo social con los niveles de depresión en personas mayores, GallardoPeralta, Sánchez-Moreno, Arias-Astray y Lópezde-Roda (2015) también encontraron que la reciprocidad en el apoyo entre cónyuges no funciona del mismo modo en el intercambio emocional y en el instrumental. Mientras que el apoyo instrumental procedente del cónyuge disminuye la depresión en el receptor, el apoyo emocional recíproco se relaciona con niveles de depresión más elevados. La explicación a este hecho podría apuntar a que dicha reciprocidad puede no ser entendida por las personas mayores como un intercambio voluntario, sino como una imposición situacional que, en contextos de tensión, constituya una fuente de estrés añadida, una obligación que añade dificultades en las situaciones concretas. Todos estos resultados apuntarían a un patrón de asociación complejo entre el apoyo social y la salud mental, que exigiría el análisis de los determinantes del apoyo social (Gallardo-Peralta et al., 2015). Más recientemente, investigadores como Tsuboi et al. (2016) han comprobado que el apoyo recibido y el apoyo dado (tanto instrumental como emocional) entre cónyuges eran predictores significativos del bienestar, aunque el apoyo dado de tipo instrumental era mejor predictor que el apoyo instrumental recibido, para los hombres. En general, para las mujeres, el apoyo emocional (recibido y dado) entre cónyuges se relacionaba significativamente con un mejor bienestar.

En los intercambios con los hijos adultos, el patrón de bienestar también varía dependiendo del tipo de apoyo: las relaciones emocionales equitativas generan la satisfacción vital más elevada en los padres mayores, pero no hay diferencias en su nivel de bienestar subjetivo en función de la equidad de los intercambios instrumentales. Los resultados sobre el intercambio emocional apoyan la ET y refuerzan los resultados de estudios previos al insistir en el papel destacado de las relaciones intergeneracionales emocionales para el bienestar de los mayores (Ajrouch, 2007). Sin embargo, difieren de los de investigadores como Lowenstein et al. (2007) y Thomas (2010), 
quienes encontraron los niveles de satisfacción vital más elevados vinculados a la satisfacción por dar más de lo recibido. También GallardoPeralta et al. (2015) encontraron que el apoyo instrumental procedente de los hijos adultos se asocia con mayores puntuaciones en depresión, pero que el apoyo emocional equitativo se asocia con menor depresión. El hecho de que las personas mayores puedan establecer una relación equitativa de apoyo emocional con sus hijos contribuiría al desarrollo de sentimientos de autoeficacia, es decir, daría lugar a sentimientos positivos para prevenir problemas mentales. Se trataría de actuaciones exitosas -en este caso, el retribuir el apoyo- que generan una sensación de control y eficacia, sentimientos que a su vez incrementan la autoestima de las personas (Thoits, 2011) y previenen sentimientos de dependencia y aislamiento. Nuestros resultados, por tanto, indican que cuando se controla el estado funcional, el mayor bienestar subjetivo proviene del intercambio emocional equitativo con los hijos adultos, aunque el tamaño del efecto pequeño relativiza este hallazgo en cierta medida.

También con los hermanos, la equidad desempeña un papel diferente dependiendo del tipo de intercambio: mientras que para la ayuda instrumental los intercambios equitativos generan el mayor bienestar subjetivo, seguidos de aquellos en los que las personas mayores dan más de lo que reciben, en el apoyo emocional los intercambios equitativos y no equitativos con los hermanos producen el mismo nivel de bienestar. Lo importante para la persona mayor no parece ser el balance final, sino la existencia de hermanos con los que intercambiar apoyo emocional. Este patrón de intercambio con los hermanos difiere de los resultados de Thomas (2010), quien obtuvo que recibir más apoyo de los hermanos conducía a mayor bienestar en las personas mayores que darlo.

Con los amigos, tanto en las relaciones de apoyo instrumental como emocional, además de los intercambios equitativos, aquellos en que la persona mayor da más apoyo del que recibe, producen los niveles más elevados de satisfacción vital. La capacidad de proporcionar apoyo a los amigos y el sentimiento de autonomía generado pueden estar asociados a esos niveles de satisfacción vital elevados en la vejez. Además, existen normas importantes en las relaciones de rol con los amigos para darles apoyo, lo que puede reforzar sus identidades de rol y promover su bienestar (Thomas, 2010). Este resultado va en la línea de otros hallazgos previos indicando que la provisión de apoyo tiene efectos positivos sobre el bienestar psicológico al potenciar el control personal (Liang, Krause, \& Bennet, 2001) y al aumentar el afecto positivo entre amigos ( $\mathrm{Li}$ et al., 2014).

Finalmente, la satisfacción vital de las personas mayores no se asocia con la presencia o ausencia de equidad en las relaciones mantenidas con los vecinos. En relaciones secundarias como estas en la vida de los mayores, la equidad o no equidad del intercambio no contribuye diferencialmente a la satisfacción vital. Variables relacionadas con la integración en la comunidad han demostrado su importancia más allá de la reciprocidad en el intercambio (Thomése, van Tilburg, \& Knipscheer, 2003). Gracia y Herrero (2006) también obtuvieron un efecto positivo de la percepción de apoyo comunitario en el ajuste y bienestar de las personas mayores, sin tener en cuenta cuestiones de reciprocidad. Asimismo, Greenfield y Reyes (2015) encontraron pocas asociaciones entre relaciones con los vecinos y afecto positivo o negativo en la vejez.

Además, como algunos autores han apuntado (p. ej., Väänänen, Buunk, Kivimäki, Vahtera, $\&$ Koskenvuo, 2008), es importante tener en cuenta las diferencias de género en la percepción del apoyo dado y recibido y su impacto sobre el bienestar. Aunque no se han analizado los datos desde esta perspectiva, estudios previos han encontrado que las mujeres son más sensibles que los hombres al apoyo emocional recibido de las personas más cercanas, y que el riesgo para su bienestar mental se reduce en mayor medida que en los hombres cuando reciben apoyo (Ang \& Malhotra, 2016; Kendler, Mayers, \& Prescott, 2005; Neff \& Karney, 2005).

Varias limitaciones del presente trabajo deben indicarse. Primero, la naturaleza transversal de las asociaciones entre equidad percibida y satisfacción vital puede reflejar sesgos de 
respuesta en algunas personas que podrían estar predispuestas a evaluaciones pesimistas de sus intercambios familiares y sociales y de su satisfacción vital. Segundo, solo se ha calculado la equidad a partir de las respuestas de una de las partes. Falta conocer la visión del otro miembro de la díada de intercambio para conseguir una imagen más completa sobre la relevancia de la reciprocidad en las diferentes relaciones interpersonales en la vejez. Tercero, es necesario investigar en el futuro las características específicas del intercambio de apoyo que mejoran el bienestar de quien lo proporciona. El trabajo de Morelli, Lee, Arnn y Zaki (2015) sugiere que apoyo instrumental y apoyo emocional generan efectos interactivos sobre el bienestar, siendo la involucración emocional de quien proporciona el apoyo lo que determina que el apoyo instrumental aumente el bienestar, tanto de quien da el apoyo como de quien lo recibe. Considerar esta variable en los trabajos sobre fuentes y tipos de apoyo social en la vejez favorecerá progresar en el conocimiento sobre los determinantes del bienestar de los proveedores y receptores de apoyo.

De forma absoluta, la equidad caracteriza la mayoría de los intercambios de apoyo de las personas mayores con sus redes familiares y sociales. Los intercambios equitativos con la familia se relacionan positivamente con la satisfacción vital en la vejez, aunque la naturaleza emocional o instrumental del apoyo dentro del matrimonio, con los hijos y con los hermanos matiza esa relación, con tamaños del efecto pequeños en todos los casos. Con los amigos, tanto la equidad como dar más de lo que se recibe, genera los niveles más elevados de bienestar subjetivo; cuando el intercambio no es equitativo, para sentirse bien en la vejez es mejor dar que recibir. El estudio también enfatiza la importancia de diferenciar la naturaleza del apoyo intercambiado por las personas mayores, y la necesidad de seguir investigando sobre distintos tipos de solidaridad en los sistemas informales de apoyo, dada su participación diferencial en el bienestar en la vejez.

\section{Agradecimientos}

Financiación: Investigación realizada con el apoyo de la Consejería de Educación de la Junta de Castilla y León al proyecto SA015A08.

\section{Referencias}

Ahmed-Mohamed, K., Fernández-Mayoralas, G., Rojo-Pérez, F., Forjaz, M. J., \& MartínezMartín, P. (2013). Perceived social support of older adults in Spain. Applied Research Quality Life, 8, 183-200. doi: 10.1007/ s11482-012-9184-8

Ajrouch, K. J. (2007). Health disparities and Arab-American elders: Does intergenerational support buffer the inequality-health link? Journal of Social Issues, 63(4), 745-758. doi:10.1111/ j.1540-4560.2007.00534.x

Ang, S., \& Malhotra, R. (2016). Association of received social support with depressive symptoms among older males and females in Singapore: Is personal mastery an inconsistent mediator? Social Science and Medicine, 153, 165-173. doi: 10.1016/ j.socscimed.2016.02.019

Armi, F., Guilley, E., \& Lalive d'Epinay, C. J. (2008). Health: Support provided and received in advanced old age. Zeitschrift für Gerontologie und Geriatrie, 41, 56-62. doi:10.1007/s00391-007-0457-z

Boerner, K., \& Reinhart, J. P. (2003). Giving while in need: Support provided by disabled older adults. Journal of Gerontology: Social Sciences, 58B(5), S297-S304.

Buz, J. (1996). Mini GDS-8: una nueva versión breve para ancianos institucionalizados. Geriátrika, 12, 41-45.

Buz, J., Mayoral, P., Bueno, B., \& Vega, J. L. (2004). Factores sociales del bienestar subjetivo en la vejez. Revista Española de Geriatría y Gerontología, 39(3), 23-29.

De Jong Gierveld, J., \& Perlman, D. (2006). Long-standing nonkin relationships of older adults in the Netherlands and the 
United States. Research on Aging, 28(6), 730-748. doi:10.1177/0164027506291873

Deeg, D. J. H., Beekman, A. T. F., Kriegsman, D. M. W., \& Westendorp-de Seriere, M. (Eds.). (1998). Autonomy and well-being in the aging population 2. Report from the Longitudinal Aging Study Amsterdam 1992-1996. Amsterdam: VU University Press.

DePaulo, B. M., \& Morris, W. L. (2005). Singles in society and in science. Psychological Inquiry, 16, 57-83. doi:10.1080/1047840X.2005.9682918

Diener, E. (2009). SWLS translations. Champaign, IL: University of Illinois at Urbana-Champaign. Recuperado de http:// s.psych.uiuc.edu/*ediener/SWLS.html

Diener, E., Emmons, R., Larsen, R. J., \& Griffin, S. (1985). The Satisfaction with Life Scale. Journal of Personality Assessment, 49(1), 71-75. doi:10.1207/s15327752jpa4901_13

Dowd, J. (1975). Aging as exchange: A test of the distributive justice proposition. The Pacific Sociological Review, 21, 351-375. doi:10.2307/1388852

Fernández-Ballesteros, R. (2002). Social support and quality of life among older people in Spain. Journal of Social Issues, 58, 645-659. doi:10.1111/1540-4560.00282

Fingerman, K. L., Kim, K., Tennant, P. S., Birditt, K. S., \& Zarit, S. H. (2015). Intergenerational support in a daily context. The Gerontologist, 1-33. doi:10.1093/geront/gnv035

Folstein, M. F., Folstein, S. E., \& McHugh, P. R. (1975). "Mini-mental state". A practical method for grading the cognitive state of patients for the clinician. Journal of Psychiatric Research, 12, 189-198.

Fyrand, L. (2010). Reciprocity: A predictor of mental health and continuity in elderly people's relationships? A review. Current Gerontology and Geriatrics Research, 1. doi:10.1155/2010/340161

Gallardo-Peralta, L. P., Sánchez-Moreno, E., Arias-Astray, A., \& López-de-Roda, A. B. (2015). Elementos estructurales de la red social, fuentes de apoyo funcional, reciprocidad, apoyo comunitario y depresión en personas mayores en Chile. Anales de Psicología, 31(3), 1018-1029. doi:10.6018/analesps.31.3.172861

Gracia, E., \& Herrero, J. (2006). La comunidad como fuente de apoyo social: evaluación e implicaciones en los ámbitos individual y comunitario. Revista Latinoamericana de Psicología, 38(2), 327-342.

Greenfield, E. A., \& Reyes, L. (2015). Continuity and change in relationships with neighbors: Implications for psychological well-being in middle and later life. Journals of Gerontology Series B: Psychological Sciences and Social Sciences, 70(4), 607-618. doi: 10.1093/ geronb/gbu084

Grundy, E. (2005). Reciprocity in relationships: Socio-economic and health influences on intergenerational exchanges between Third Age parents and their adult children in Great Britain. The British Journal of Sociology, 56, 233-255. doi:10.1111/ j.1468-4446.2005.00057.x

Hagedoorn, M., Van Yperen, N. W., Coyne, J. C., van Jaarsveld, C. H. M., Ranchor, A. V., van Sonderen, E., \& Sanderman, R. (2006). Does marriage protect older people from distress? The role of equity and recency of bereavement. Psychology and Aging, 21(3), 611-620. doi:10.1037/0882-7974.21.3.611

Hendrix, M., Baumgartner, T., Kirschbaum, C., \& Ehlert, U. (2003). Social support and oxytocin interact to suppress cortisol and subjective responses to psychosocial stress. Biological Psychiatry, 54, 1389-1398. doi:10.1016/S0006-3223(03)00465-7

Ikkink, K. K., \& van Tilburg, T. (1999). Broken ties: Reciprocity and other factors affecting the termination of older adults' relationships. Social Networks, 21, 131-146. doi:10.1016/S0378-8733(99)00005-2

Johar, M., Maruyama, S., \& Nakamura, S. (2015). Reciprocity in the formation of intergenerational coresidence. Journal of Family and Economic Issues, 36, 192-209. doi: 10.1007/s10834-013-9387-7

Kendler, K. S., Mayers, M. S., \& Prescott, C. A. (2005). Sex differences in the 
relationship between social support and risk for major depression. A longitudinal study of opposite-sex twin pairs. American Journal of Psychiatry, 162, 250-256. doi: 10.1176/ appi.ajp.162.2.250

Kim, H. K., Hisata, M., Kai, I., \& Lee, S. K. (2000). Social support exchange and quality of life among the Korean elderly. Journal of Cross-Cultural Gerontology, 15, 331-347. doi:10.1023/A:1006765300028

Lee, H. J., Lyu, J., Lee, C. M., \& Burr, J. A. (2013). Intergenerational financial exchange and the psychological well-being of older adults in the Republic of Korea. Aging and Mental Health. Publicación anticipada en línea. doi:10.1080/13607863.2013.784955

Lee, K. S., \& Ono, H. (2012). Marriage, cohabitation and happiness. A crossnational analysis of 27 countries. Journal of Marriage and Family, 74(5), 953-972. doi:10.1111/j.1741-3737.2012.01001.x

Leopold, T. \& Raab, M. (2013). The temporal structure of intergenerational exchange: A within-family analysis of parent-child reciprocity. Journal of Aging Studies, 27, 252-263. doi: 10.1016/j.jaging.2013.05.001

Li, T., Fok, H. K., \& Funk, H. H. (2011). Is reciprocity always beneficial? Age differences in the association between support balance and life satisfaction. Aging $\mathcal{E}$ Mental Health, 15(5), 541-547. doi:10.1080/13607863.2010.551340

Li, H., Ji, Y., \& Chen, T. (2014). The roles of different sources of social support on emotional well-being among Chinese elderly. PLoS One, 9(3), e90051. doi: 10.1371/jounal.pone.0090051

Liang, J., Krause, N. M., \& Bennett, J. M. (2001). Social exchange and wellbeing: Is giving better than receiving? Psychology and Aging, 16(3), 511-523. doi:10.1037/0882-7974.16.3.511

Lowenstein, A., \& Daatland, S. (2006). Filial norms and family support in a comparative cross-national context: Evidence from the OASIS study. Ageing and Society, 26, 203-223. doi:10.1017/ S0144686X05004502
Lowenstein, A., Katz, R., \& Gur-Yaish, N. (2007). Reciprocity in parent-child exchange and life satisfaction among the elderly: A cross-national perspective. Journal of Social Issues, 63, 865-883. doi:10.1111/ j.1540-4560.2007.00541.x

Lowenstein, A., Katz, R., \& Gur-Yaish, N. (2008). Cross national variations in elder care: Antecedents and outcomes. En M. E. Szinovacz \& A. Davey (Eds.), Caregiving contexts (pp. 93-115). Nueva York: Spring Publishing.

Lu, N., Liu, J., \& Lou, V. (2016). Exploring the reciprocal relationship between caregiver burden and the functional health of frail older adults in China: A cross-lag analysis. Geriatric Nursing, 37, 19-24. doi: 10.1016/ j.gerinurse.2015.08.014

McMunn, A., Nazroo, J., Wahrendorf, M., Breeze, E., \& Zaninotto, P. (2009). Participation in socially-productive activities, reciprocity and wellbeing in later life: Baseline results in England. Ageing and Society, 29, 765-782. doi:10.1017/ S0144686X08008350

Meléndez-Moral, J. C., Tomás-Miguel, J. M., \& Navarro-Pardo, E. (2007). Análisis de las redes sociales en la vejez a través de la entrevista Manheim. Salud Pública de México, 49(6), 408-414.

Morelli, S. A., Lee, I. A., Arnn, M. E., \& Zaki, J. (2015). Emotional and instrumental support provision interact to predict well-being. Emotion, 15(4), 484-493. doi:10.1037/emo0000084

Neff, L. A., \& Karney, B. R. (2005). Gender differences in social support: A question of skill or responsiveness? Journal of Personality and Social Psychology, 88, 79-92. doi:10.1037/0022-3514.88.1.79

Nyqvist, F., Forsman, A. K., Giuntoli, G., \& Cattan, M. (2013). Social capital as a resource for mental well-being in older people: A systematic review. Aging and Mental Health, 17(4), 394-410. doi:10.1080/13607863.2012.742490

Sánchez, M. M., de Jong Gierveld, J., \& Buz, J. (2014). Loneliness and the exchange 
of social support among older adults in Spain and the Netherlands. Ageing and Society, 34(2), 330-354. doi:10.1017/ S0144686X12000839

Shaw, B. (2005). Anticipated support from neighbors and physical functioning during later life. Research on Aging, 27(5), 503-525. doi: $10.1177 / 0164027505277884$

Silverstein, M., Conroy, S. J., Wang, H., Giarrusso, R., \& Bengtson, V. L. (2002). Reciprocity in parent-child relations over the adult life course. Journal of Gerontology: Social Sciences, 57B, S3-S13.

Thoits, P. A. (2011). Mechanisms linking social ties and support to physical and mental health. Journal of Health and Social Behavior, 52, 145-161. doi:10.1177/0022146510395592

Thomas, P. A. (2010). Is it better to give or to receive? Social support and the well-being of older adults. Journals of Gerontology Series B: Psychological Sciences and Social Sciences, 65B, 351-357. doi:10.1093/geronb/gbp113

Thomése, F., van Tilburg, T. G., \& Knipscheer, K. (2003). Continuation of exchange with neighbors in later life: The importance of the neighborhood context. Personal Relationships, 10, 535-550. doi:10.1046/ j.1475-6811.2003.00064.x

Tsuboi, H., Hirai, H., \& Kondo, K. (2016). Giving social support to outside family may be a desirable buffer against depressive symptoms in community-dwelling older adults: Japan gerontological evaluation study. BioPsychoSocial Medicine, 10, 18. doi: 10.1186/s13030-016-0064-6

Väänänen, A., Buunk, P. A., Kivimäki, M., Vahtera, J., \& Koskenvuo, M. (2008). Change in reciprocity as a predictor of depressive symptoms: A prospective cohort study of Finnish women and men. Social Science and Medicine, 67, 1907-1916. doi: 10.1016/j.socscimed.2008.09.015

van Tilburg, T. G. (1992). Question sequence effects in the measurement of reciprocity. Quality Eु Quantity, 26(4), 395-408. doi:10.1007/BF00170451 van Tilburg, T. G., \& Broese van Groenou, M. I. (2002). Network and health changes among older Dutch adults. Journal of Social Issues, 58, 697-713. doi:10.1111/1540-4560.00041

Voorpostel, M., Lippe, T., Dykstra, P. A., \& Flap, H. (2007). Similar or different? The importance of similarities and differences for support between siblings. Journal of Family Issues, 28(8), 1026-1053. doi:10.1177/0192513X07300713

Walster, E., Walster, G. W., \& Berscheid, E. (1978). Equity: Theory and research. Boston, MA: Allyn \& Bacon.

\section{Notas}

* Artículo de investigación. 